\title{
Urgences
}

\section{Éthopée : une autre historiographie pour une autre Histoire sans Sujet}

\section{Danielle Lafontaine}

Numéro 21, novembre 1988

Bagatelles et crases

URI : https://id.erudit.org/iderudit/025495ar

DOI : https://doi.org/10.7202/025495ar

Aller au sommaire du numéro

Éditeur(s)

Urgences

ISSN

0226-9554 (imprimé)

1927-3924 (numérique)

Découvrir la revue

Citer ce document

Lafontaine, D. (1988). Éthopée : une autre historiographie pour une autre Histoire sans Sujet. Urgences, (21), 77-86. https://doi.org/10.7202/025495ar d'utilisation que vous pouvez consulter en ligne.

https://apropos.erudit.org/fr/usagers/politique-dutilisation/ 


\section{DANIELLE LAFONTAINE}

\section{Éthopée: d'une autre historiographie pour une autre Histoire sans Sujet.}

A Bernardine Jouin et Jean-Baptiste de la Fontaine ${ }^{1}$, ordinaire de la Musique du roi, restés là-bas, et aux déportés de Louisianne et d'ailleurs.

Nous ne sommes à l'aube d'aucun virage radical

de l'Histoire, d'aucun commencement absolu,

d'aucune rupture définitive

marquant la naissance inaugurale

d'un nouveau monde ou d'une nouvelle culture.

Et pourtant dans cette reconnaissance

des pesanteurs passées

comme de l'absence de fatalité historique

se profile peut-être quelque chose de neuf ${ }^{2}$.

Notre moment ${ }^{3}$ serait celui de la construction délibérée

d'unités de vie artificielles ${ }^{4}$, d'fles

se complétant et obéissant à leur rythme.

... le mode de reproduction qui prend corps vise a reterritorialiser. Pour y parvenir, une seule méthode: métisser, proportionner, enraciner. Le métissage à l'horizontale, de ce qui a été séparé à la verticale, par le remembrement des travaux, des savoirs, des moyens de production destinés à être renouvelés, constitue le premier pas vers la généralisation de telles unités.

Serge Moscovici (1976)

... ce point de vue... nous oblige à être extrêmement attentif aux pratiques qui combattent, par exemple, la dissociation et la séparation des générations, des savoirs, des temps et des lieux que des hommes et des femmes ont marqués. Marcel Rioux (1982) ${ }^{6}$

Au métissage des savoirs

garantissant la production des ces unités, donnons le nom d'Éthopée.

C'est dans le second tome du Dictionnaire universel «contenant généralement tous les mots 
françois tant vieux que modernes et les termes de toutes les sciences et les arts», recueilli et compilé par feu Meffire Antoine Furetière et publié à la Haye et Rotterdam chez Arnout et Reinier Leers en 1690 , que l'on peut trouver la définition suivante: «Éthopée. $f$. . figure de rhétorique, qui est une defcription des moeurs et des paffions de quelques perfonnes, qui s'appelle auffi Éthologie. Elle diffère de la profopée, en ce que celle-cyce sont des perffonnes feintes qu'on fait parler; au lieu que dans l'éthopée on défcrit les moeurs des perfonnes véritables. Ce mot eft $\mathrm{Grec}$, et fignifie peinture des moeurs. Il vient du Grec Ithos, mos, confuetudo, et poieo, facio, fingo, defcribo.»

Nous ferons parler les vivants et les morts.

Nous tracerons de singuliers tableaux.

Micmacs de Gespeoeg appauvris du commerce des pelletries, morts de faim dans la neige;

habitants des postes de pêche de Percé et Pabos dévastés par les soldats de Wolfe en 1758; familles acadiennes traquées dans les bois de la Baie-des-Chaleurs après la dispersion de 1755;

noyés irlandais du Carrik aux corps déchiquetés sur les côtes basses du cap des Rosiers, votre Rassemblement a déjà commencé.

S'ouvre un temps raisonnable où les âmes des mariés seront dénudées par leurs descendantes mêmes.

Dans nos surprenantes machines nous remonterons les aires et les périodes à la découverte de certaines isles et pays oul'on dit qu'il se doib trouver riches choses $^{7}$, là où l'on se chauffe, epsegeneg; plus loin qu'au Grand Banc de Miscou; en direction du lieu médian ${ }^{8}$ d'avant le nom fondant le pouvoir de parler 
et rendant possible tout échange;

jusqu'au berceau des formes constatables.

Chargeons nos biscuits, cidre, bière, sapinette; préparons à l'avance grave et vigneaux; lavons les galets, tendons la Grande Oreille:

Oteepayamsoowuk,

Métis en charge de nous-mêmes.

Et les récits combien exotiques

de nos fascinantes expéditions archéologiques en surprendront plusieurs.

Car nos listes et nos taxinomies, sous le soleil brûlant de l'événement, hâtivement constituées, pourront dérouter;

nos hypothèses inquiéter l'invisible college.

Qu'importe, si ceux et celles qui ont la parole parviennent à questionner le mode d' existence des réalités humaines et sociales territorialisées comme il doit l'être

pour que soient envisagées et construites ces îles assurant la pérennité de l'espèce.

Qu'importe, si nos troublants récits ouverts des moeurs et passions datés et situés

des personnes véritables

nous laissent mieux entrevoir

l'éblouissante profondeur

de l'enracinement historique, et deviner la fulgurante complexité des enchainements et des causes raccordant dans le temps et l'espace plusieurs séries de faits. 
Peinture de moeurs aux ysles de Gachepé et ailleurs.

François Belcourt de Lafontaine, marié à Grande-Rivière, le 15 novembre 1783 à Charlotte Dupuis, veuve Beaudin, son seul fils François, uni à Grande-Rivière le 18 février 1822 à Suzanne Beseau, puis son petit-fils François marié au même endroit le 21 février 1846 à Nathalie Béliveau, et son arrière petit-fils Sévère, né à Grande-Rivière le 15 septembre $1849^{10}$ comprirent-ils le rôle du capital marchand anglo-normand dans l'organisation de l'économie morutière gaspésienne au 19ième siècle?

Employés locaux de la puissante société Charles Robin and Co., de père en fils engagés dans la production de morue séchée destinée à l'exportation, devinèrent-ils l'existence de ces vastes systèmes d'échanges en train de se mettre en place? Surent-ils ce qu'il advenait de ces précieuses marchandises vendues à Rio ou à Naples, et plus encore du profit constitué au fur et à mesure de leur mouvement dans l'espace maritime et terrestre? Eurent-ils cette indispensable «imagination» selon Alfred Marshall nécessaire à la saisie de ces «causes des événements visibles... qui demeurent cachées ou enfouies sous la surface» ${ }^{11}$ ?

Furent-ils parmi les signatairès d'une pétition envoyée le 30 janvier à l'Assemblée législative du Bas-Canada et intitulée «Pétition des habitants du comté de Gaspé se plaignant de divers griefs», dans laquelle l'on reprochait à la compagnie Robin «son influence indue sur les habitants qui lui sont presque tous endettés» 12 ?

Isaac, le fils de Sévère ne pourra quant à lui ni lire ni écrire et son fils Charles-Édouard, né à Grande-Rivière le 9 mars 1907 ne fréquentera l'école qu'une année, vivra quelques années à Saint-Omer dans la Baiedes-Chaleurs, avant d'être embauché à treize ans par une compagnie de bois, pour porter l'eau aux bûcheurs qui passent l'hiver au campe. Il y sera ensuite cook et enfin charretier transportant sur un traîneau tiré par des chevaux les arbres débités jusqu'à la rivière en vue de la drave qui les acheminera par flottage vers les scieries au bord de la mer.

À seize ans Charles-Édouard quitte pour toujours la Gaspésie en direction de Montréal. Il y rejoint sa grand-mère de Lafontaine, une irlandaise née Sutton qui l'appellera $N e d$, et son oncle paternel, débardeur au Port de Montréal qui l'aide à obtenir un travail régulier $a u$-bord-de-l'eau et l'initie aux rudiments de la boxe professionnelle. 
Débardeur durant cinquante ans Charles-Édouard manipule et voit circuler des tonnes de marchandises d'importation et d'exportation: viandes, grain, charbon, farine, thé, café, babiolles venues des quatre coins du monde. Toutes ces années il trouve dans la boxe qu'il pratique, et pour laquelle il s'entraîne, puis qu'il enseigne, une joie profonde.

Il éprouve une grande réticence à évoquer tant ses origines gaspésiennes pourtant incrites dans sa langue parlée, que l'existence définie de ses ancêtres dont il ne conserve que des souvenirs vagues: la grand-mère de Lafontaine se battant à mains nues en anglais avec les caribous dans les Hautes; le frère de la grand-mère Bélanger, une micmac née Guédry, vivant dans son wigwam avec les rats à l'entrée du village; l'oncle revenu des États avec une montre en or Waltham; les vieux racontant aux veillées que les Lafontaine se seraient jadis fait volé 1'île d'Anticosti.

Sans livres et sans archives dont il ignore jusqu'à l'existence, comment Charles-Édouard eut-il pu savoir que François Belcourt de la Fontaine, premier des siens installé à Grande-Rivière, était né en 1746 à Québec et qu'il était le fils cadet de Jacques de la Fontaine, français débarqué à Québec le 28 août 1726 avec le marquis de Beauharnois dont il était secrétaire, et de Charlotte Bissot de Vincennes née à Québec en $1704^{13}$ ?

Quel eut pu être pour lui l'intérêt à se souvenir de tous ces noms, de tous ces morts depuis aussi longtemps disparus, l'intérêt à se représenter leurs existences particulières pensées indépendantes de la sienne, leurs actions, leurs joies ou leurs malheurs estimés sans rapports avec les siens?

La grande crise de 1929 et les diverses récessions qui suivirent, sa mobilisation à Halifax durant la deuxième guerre mondiale sous le nom plus court de Lafontaine, quelques longues grèves au Port de Montréal et la crainte quasi permanente de la maladie qui laisse alors des dettes impossibles à rembourser, le préoccupent bien davantage.

Il habite un quartier ouvrier exclusivement canadien-français de l'Est de Montréal, mais il dit que ce sont «les anglais qui mênent» partout au Québec et ailleurs, parce qu'ils ont de l'argent, et que l'argent «contôle le monde» de toute éternité. Il sait que les canadiens-françạis «étaient là» avant les anglais, mais il ignore tout de la capitulation.

Il ne sait pas que Jacques de Lafontaine Belcour fut durant quatre années avec M. Cugnet, comme le rapportait en 1919 Pierre-Georges Roy dans son ouvrage consacré à la famille De Víncennes ${ }^{14}$, l'un des deux 
aviseurs légaux francophones du Conseil de Guerre établi dans le gouvernement de Québec par le général Murray, le 31 octobre 1760 «conformément à l'article quarante-deux de la capitulation générale de (cette) colonie...» (Régistre de la Cour Militaire, cahiers 1, p. 3, cité p. 186)

Avant de rendre leurs jugements, raconte Roy ${ }^{15}$, les membres du Conseil militaire entendaient l'un ou l'autre des aviseurs qui les «renseignaient sur les anciennes lois françaises, nos coutumes, les décisions des anciens tribunaux...» Mais poursuit Roy:

La conquête du Canada par les armes anglaises amena ici nombre de négociants amplement pourvus de capitaux qui ne demandaient pas mieux que de prendre à leur compte les exploitations qui jusque-là avaient été aux mains des négociants français et canadiens. Le Labrador avec ses réserves inépuisables ne tarda pas à attirer leur attention. Dès 1761, le gouverneur Murray, sans s'occuper des droits des héritiers Bissot, commença à concéder de larges portions de la seigneurie de Mingan à M. Isbister (p. 190)

Roy rapporte que le 23 octobre 1763, M. de Lafontaine de Belcour adressait une longue lettre au comte de Halifax, secrétaire d'État des colonies, et dans laquelle il écrivait, en français: «La qualité de sujet de Sa Majesté Britannique en Canada, me fait prendre la liberté de réclamer la protection de votre excellence sur les injustices qui me sont faites ainsi qu'à ma famille...» La lettre était accompagnée de deux mémoires tentant d'établir les droits des héritiers Bissot de Vincennes et Jolliet au poste et aux îles Mingan ${ }^{16}$. Le 24 avril 1764, le gouverneur Murray faisait en réponse aux représentations de Jacques de Lafontaine, parvenir une longue lettre au comte d'Halifax et dans laquelle il écrivait notamment, en anglais :

That I did recommend Mr. Isbister to Mr. Lafontaine \& Mr. Tachet as the most proper man for the post of Mingan is most certain; this Mr. Isbister had been long Principle Governor at Hudson Bay, he came here in the year 1760 with a wife and six small children, had merchandises to the amount of nine hundred Pounds ${ }^{17}$.

Au sujet de Lafontaine, Murray écrivait:

...had he behave properly he might still have possessed that Post, but that very autumn 1761 in his way to another Post of his Montagamiou, his conduct confirmed every bad thing that I had heard of him, and in place of being his Protector, I ever after looked upon him as an enemy of the State. Mr. Isbisters letter of March 1762 
(...) evince (...) how faulty I should have been to have countenanced such a man. This behaviour of Mr. Lafontaine and the Intelligence we had that winter of Privateers and Pirates, who sheltered and subsisted themselves by means of the Posts and the Bay of Chaleur made me think it highly necessary at the sollicitation of all the merchants here, to arm a vessel for the protection of their trade.. .18

Jugé ennemi de l'État, associé aux pirates de la Baie-des-Chaleurs nuisant au commerce des marchands, de Lafontaine traité de «scélérat qui fait honte à la nature humaine» par Murray, meurt à Québec le 18 juin 1765. Sa propriété est quelque temps plus tard «vendue à l'enchère et adjugée par un décret de la cour des Plaidoyers communs, le 13 avril 1766 au lieutenant Malcolm Fraser qui la vendit le 22 octobre 1767 à Samuel Holland, arpenteur-général de la province du Bas-Canada.» 19

Le plus jeune de ses fils, François, né le 22 décembre 1746 alors que sa mère Charlotte Bissot de Vincennes a déjà 42 ans et mourra trois ans plus tard, épouse à Grande-Rivière en 1782, Charlotte Dupuis, veuve Beaudin, sa voisine plus âgée que lui.

Parmi les premiers habitants à résider en permanence, après la Conquête, à l'embouchure de la Grande Rivière, les de Lafontaine et les Beaudin occupent des lots adjacents parmi les plus favorables à la transformation et au séchage de la morue.

Belcourt de Lafontaine, son fils François, né en 1786, et son petit-fils François, né en 1821 seront maîtres de grave, dealer ou «pratique» au service de la compagnie Charles Robin and Co. seigneur de GrandeRivière.

«C'est en juin 1793, écrivent les auteurs de l'Histoire de la Gaspésie ${ }^{20}$, que Robin achète pour 100 livres sterling les droits de ce fief, coupant l'herbe sous le pied à un certain François de La Fontaine de GrandeRivière, qui était venu solliciter de la compagnie de Jersey un prêt de 100 livres pour cet achat». La compagnie pourrait de ce fait exiger des habitants censitaires de la localité une rente seigneuriale proportionnelle au nombre de barges utilisées dans leur établissement de pêche.

Deux ans auparavant, en 1791, l'achat de la seigneurie, également convoitée par la Mathew Steward and Co., au marchand britannique Duncan Anderson $^{21}$, avait donné lieu à des échanges de lettres. Le 6 septembre Charles Robin écrivait, en anglais: 
One Mr. de la Fontaine has been upon bargain with the proprietor of the Seigneurie of Grand River (...) but the proprietor will not hearken to anything except I become guarantee for the payment (...). De la Fontaine has applied to me (...) but if you will take my advice we shall buy it immediately (...). Throwing it in La Fontaine way would be injuring ourselves amasingly. I have put it off for this fall by telling him that I must consult you on the matter and that I would let him know the result next spring. Therefore it would be proper you wrote to me in french acquainting me that you could not advance the money ...22

Propriétaire formel d'un établissement de pêche situé certes à proximité d'une bonne grave pour sécher la morue, mais dont la valeur reste entièrement réglée sur celle que le marché assigne au poisson, François Belcourt de la Fontaine serait comme ses voisins un petit-producteur endetté à qui la compagnie Robin prêterait à crédit des ressources indispensables à la mise en valeur du poisson: agrès, échafaud, outils nécessaires à l'extraction ou à la transformation de la marchandise.

Si la «molue» coûte cher à produire, elle ne rapporte pas beaucoup aux petits-producteurs et le déséquilibre entre les valeurs inscrites au débit et au crédit se creuse d'années en années, rendant tout épargne, comme toute liquidation de dette, impossibles.

Pour pouvoir maintenir la production et conserver l'établissement ainsi que les biens meubles hypothéqués, il faut à tout prix investir et donc emprunter à nouveau auprès de la compagnie en voyant du même coup augmenter la valeur annuelle de sa dette transmise intégralement aux descendants.

Entre les naissances de François, en 1786 et celle de Sévère en 1849, la dette de la famille de la Fontaine envers la compagnie Robin n'allait cesser de s'alourdir, la dépossession de devenir plus intégrale.

Certes jusqu'au début du XXième siècle les générations successives restent $d$ leur place, sur le lot 6 de la Grande-Rivière et reproduisent immuablement leurs manières de vivre et de produire. Mais c'est tout le reste du monde qui bouge et anime, comme une immense nappe d'ombre, le socle même des pratiques ancestrales, leur imposant un ordre de représentations dont la teneur échappe au contrôle des habitants.

Est-ce par hasard ou pour échapper à cette mouvance et aux forces qui en commandent l'agitation, que Charles-Édouard, occupera un demisiècle durant le même emploi au-bord-de-l'eau, se refusant avec 
obstination à passer contremaître, lui qui sait pourtant mieux que personne comment manier le crochet, répartir, fixer et balancer les charges au fond des navires pour éviter ces terribles accidents qui tuent ou mutilent à tout jamais débardeurs et matelots?

Est-ce sans raisons ou pour maintenir vivace une liberté du corps et de la pensée depuis longtemps soumise qu'il recueille l'hiver chez lui ces jeunes noirs de Harlem à qui il apprend patiemment à frapper juste et à recevoir des coups en leur parlant de cette discipline essentielle aux «vrais bons boxeurs»?

D'où, de quel ensemble de raisons oubliées, venues du fond des âges et transmises de générations en générations, cette vie-là, cette histoire à nulle autre pareille, tire-t-elle son élan, en même temps que son ordre tellement particulier, si peu arbitraire, qu'on la dirait après coup destin?

Quelle historiographie responsable nous en fabriquera une représentation sans s'occulter elle-même en tant qu'instrument de production et sans une élimination de larges pans de l'expérience historique?

Quels récits nous apprendront les règles complexes suivant lesquelles des liens humains d'échange sont sans cesse créés et médiés, nous donneront accès à l'intelligibilité d'une historicité générale de la condition humaine?

L'établissement de réciprocités surgit probablement dès les premiers mois de l'existence de tous et chacun, comme un problème central; un problème lié au besoin d'air, de nourriture; un problème devant absolument être surmonté et débouchant à partir de l'expérience de la satisfaction ou du manque, sur la construction, en même temps que sur l'usage effectif des catégories langagières permettant de nommer et juger ces objets ou ces personnes associés peu à peu à la satisfaction et au plaisir et servant progressivement à désigner le «bon», puis le «beau» ou le «vrai», À l'urgence vitale, comme le suggérait déjà la sémiotique pragmatique de C.S. Peirce, correspondrait donc un travail psycho-langagier débouchant sur l'élaboration des catégories d'objets bons et peut-être non-bons, sur la constitution de processus de classement, opérations rationnelles fondamentales que C. Lévi-Strauss inscrivait à l'enseigne de la «logique éternelle» du langage humain ${ }^{23}$.

Tel pourrait être le socle invariant et universel d'une culture humaine aux formes variables, trop longtemps normativement évaluées les unes par rapport aux autres et hiérarchisées verticalement. 
Tel pourrait être le lieu d'une historicité (et non d'une Histoire ascendante linéaire) en permanence marquée par la relation entre l'échange, l'attraction et l'antagonisme: le travail, la quête du plaisir et le conflit.

Les valeurs éthiques, esthétiques et épistémiques trouvent possiblement leur fondement en cette région profonde où s'édifie pour tous et chacun, à travers l'échange de signes, une subjectivité langagière à laquelle le problème d jamais entièrement surmonté du plaisir, du désir et manque, confere sa spécificité.

Ce n'est pas de la fable dont il faut nous méfier, mais de ces récits qui donnant vie à des personnes ou des entités feintes, nous voilent des réalités ou processus existants dont la vie tires ses moyens.

Ainsi la «Société» n'a pas de «volonté» ${ }^{24}$, pas d'intentionnalité; la Technologie ne «commande» pas non plus la «Société» et cette dernière tout comme l'Histoire - n'a pas de centre d'où serait dirigé un devenir historique reposant plutôt essentiellement sur le travail pratique et symbolique de personnes existantes, seules en mesure de construire des orientations, autant que des environnements sociaux viables. 


\section{Notes}

1. Une note parue dans Criées du Châtelet, novembre 1751, p. 18 et reproduite dans les Archives de Québec, XVIII, p. 145, indiquait: «Le Sr. Luc, ordinaire de la Musique de la chapelle et chambre du roi et organiste de ladite chapelle, et Bernardine de la Fontaine sa femme laquelle était héritière de Jean-Baptiste de la Fontaine, ordinaire de la Musique du roi et de Bernardine Jouin, ses père et mère; de Jeanne de la Fontaine, fille majeure, sa soeur, et de Jacques de la Fontaine de Bellecour, conseiller au Conseil Supérieur de Québec, en Canada». On trouvera ici, comme dans les archives consultées, plusieurs variations des noms de famille: Bellecourt, Belcourt, Belcour, de la Fontaine, de La Fontaine, de Lafontaine.

2. Extrait de J.-P. Boyer et D. Lafontaine, «Nouvelles technologies communicationnelles et quête d'un nouveau contrat social», Loisir et Société, automne 1988.

3. D'après Sartre, pour son éblouissante reconstruction d'un combat de boxe servant d'armature à sa Critique de la Raison dialectique: 2, l'intelligibilité de l'histoire.

4. Inspire notamment par Herbert A. Simon, The science of the Artificial, MIT Press, (1969), 1982, qui écrit de son ouvrage qu'il «prend la forme d'une fugue dont le propos et le contre-propos, durant plusieurs décades séparés comme les deux extrémités d'un continent, seraient enfin rassemblés («woven together») dans un tout» (p. ix). Prix nobel d'économie 1978, Simon y traite du fondement de la rationalité économique en relation avec le problème de la création d'environnements sociaux et symboliques artificiels.

5. Dans «Le réenchantemenr du monde», in Alain Touraine, dir., Au-delà de la crise, Paris, 1976. En 1975-76, Moscovici s'occupait à Paris avec le sociologue Alain Touraine d'un programme de troisième cycle centré sur «l'étude interdisciplinaire du changement social et des mouvements sociaux».

6. Dans «Remarques sur les pratiques émancipatoires dans les sociétés industrielles en crise», in Collectif, Les pratiques émancipatoires en milieu populaire, Institut Québécois de Recherche sur la Culture (IQRC), 1982, p. 53. Rioux avait compris avant bien d'autres l'importance du nom.

\section{Voir Jacques Cartier, Voyages en Nouvelle-France, Montréal, Hurtubise, HMH, 1977.}

8. D'après ce que Michel Foucault nomme (dans Les Mots et les Choses: une archéologie des sciences humaines, Paris, Gallimard, 1966, p. 11) la région médiane (trad. ang. d'A. M. Sheridan Smith, Pantheon, 1970, «the middle region»). Voir note 23. Aristote, rappelle René Thom, soutenait déjà que les structures réalisées dans le monde historique se développaient de l'abstrait vers le concret et Thom d'ajouter que les structures «profondes» analysées «en surface» par les morpho-linguistes générativistes n'étaient peut-être pas si profondes et que «ce qui serait beaucoup plus intéressant, c'est la dynamique générale des structures profondes.»

9. Dans sa «romance épique» (Globe and Mail) Caesars of the Wilderness, relatant les activités de la Compagnie de la Baie d'Hudson au Canada, le joumaliste Peter C. Newman note: «Interestingly, the Cree word for Métis is oteepayamsoowuk, meaning «in charge of themselves». Althought most Métis spoke French, they had a distinct language called Bungay, described by a traveller of that time as «polyglot jabber» - a mixture of broad Scots, a smattering of Gaelic and Irish brogue and rapidly uttered French.», Penguin Book, 1987, p. 208. On y retrouve nos Nicolas Montour et Simon McTavish dont L. Desrosiers nous avait déjà tracé d'hallucinants portraits dans Les engagés du Grand-Portage. Au sujet du legs 
des activités de traite des fourrures, Newman précise: «The only remaining public monument of the fur-trade fortunes is Montréal's McGill University. James McGill had been in the fur trade but gained most of his wealth from banking, timber and land speculation», p. 17.

10. D'après P. Gallant, Les registres de gaspésie (1752-1850), Montréal, Société généalogique canadienne-française, 1961, p. 174-175. L'auteur du registre note dans son introduction: «Peu de pays au monde ont une population d'origines aussi diverses que la Gaspésie...» Je remercie ici Pierre Collin archiviste à l'UQAR pour son aide. La mention «Belcourt», n'apparaît plus que pour François, fils cadet, marié à Grande-Rivière en 1783, mais ce nom se serait transmis par les deux fils aînés de Jacques de la Fontaine et leurs descendants, certains signant à l'anglaise «Belcourt» et retranchant le de lo Fontaine rappelant une origine française.

11. Dans son fameux Principles of Economics, cité par Kurt Heinzelman dans The Economics of the Imagination, The University of Massachusetts Press, Amherst, 1980, p. 289. Dans les premiers mots de son discours de réception du prix nobel 1978, H. A. Simon rappelait que Marshall avait envisagé son Économie Politique à la fois comme une science de la richesse et comme une science de l'homme, mais qu'hélas l'économie ne s'était nor. mativement préoccupée que de certains aspects de la raison humaine, les liant à une théorie contestable et insoutenable de l' «équilibre» général des marchés. Ce texte choc est publié sour le titre: «Rational Decision Making in Business Organizations», The American Economic Review, sept. 1979.

12. Dans Journaux de l'Assemblée législative du Bas-Canada, 1830, Appendice T., cité par André Lepage dans Le capitalisme marchand et la pêche à la morue en Gaspésie. La Charles Robin and Company dans la Baie-des-Chaleurs (1820-1870), Thèse de Doctorat en anthropologie, Laval, 1983, p. 414. Mais à l'hiver 1830-31, rapporte Charles Hegel dans sa préface, G.W.F. Hegel présentait déjà en Europe sous forme de conférences, des chapitres de sa Philosophie de l'Histoire laquelle serait critiquée par K. Marx au nom d'une conception matérialiste (mais toujours linéaire et ascendante...) de l'Histoire.

13. Dans Cyprien Tangay, Dictionnaire généalogique des familles canadiennes depuis la fondation de la colonie jusqu'à nos jours, Troisième Volume, Montréal, Eusèbe Sénécal, MDCCCLXXXVII, p. 287.

14. Dans Pierre-Georges Roy, Le Sieur de Vincennes fondateur de L'Indiana et sa famille, Québec, Charrier et Dugal, 1919. D'après les Actes d'état civil relatifs aux Bissot de Vincennes fournis par Roy, Charlotte Bissot de Vincennes, épouse de Jacques de la Fontaine était la fille de Marie Dumont et de François Bissot de Vincennes de la Rivière, lui-même fils de François Bissot et de Marie Couillard, cette dernière fille de Guillaume Couillard et de Guillemette Hébert, fille de Louis, apothicaire parisien, et de Marie Rollet, établis avec leur trois enfants en Nouvelle-France en 1617. À leur sujet Marthe Faribault-Beauregard note: «Il n'existe pas de descendance mâle de notre premier défricheur. Les Hébert du Canada ont une tout autre origine. Par contre Guillemette Hébert...», dans La géréalogie, Éditions de l'Homme, Montréal, 1987, p. 67.

15. Roy, ibid, 1919, p. 190. Au lendemain de la capitulation de la colonie, le Conseil militaire de Québec remplace le Conseil supérieur dont de la Fontaine était membre. C'est le 11 avril 1735 que le Roi avait informé MM. de Beauharnois et Hocquart que des quatres places de conseillers vacantes, une avait été accordée au sieur de la Fontaine qui dans une lettre envoyée en 1732 au ministre Maurepas s'était plaint des «abus qui se sont commis jusqu'à présent dans les études de notaires de la Nouvelle-France.» Ce demier se vit ensuite délégué à Montréal de novembre 1740 à octobre 1741 pour remplir temporairement la 
charge de juge laissée vacante par la mort de M. Raimbault, voir à ce sujet E. Z. Massicotte, Bulletin des Recherches Historiques. Les juges de Montréal sous le Régime Français, 1648-1760, XXVII, p. 183. Dans une lettre au général Murray, de la Fontaine écrit à son propre sujet: «...trente années de conseiller au Conseil Supérieur dont j'étais l'ancien, 22 années Secrétaire du Gouvernement...»

16. Roy, ibid, 1919, p. 202, d'après les Archives du Canada, série Q., vol. 2. Ces deux mémoires auraient été publiés dans le factum soumis au Conseil privé dans la cause «The Labrador Company and her Majesty the Queen», pp. 303 et 305.

17. Trad. «Que j'aie recommandé $M$. Isbister à Messieurs Lafontaine et Tachet en tant qu'homme le plus approprié pour le poste de Mingan, la chose est certaine; ce Monsieur Isbister a été longtemps principal gouvemeur à la Baie d'Hudson, il est venu ici en 1760 avec une femme et six jeunes enfants, et des marchandises d'une valeur de neuf cent Livres.» Alexandre Kennedy Isbister, petit-fils d'Isbister et fils d'une Cree, employé comme ses pères de la Compagnie de la Baie d'Hudson allait démissionner de son poste, poursuivre des études à l'Université d'Aberdeen avant de se porter à la défense des Métis de la Rivière Rouge «traités en esclaves comme la population noire de certains états américains» par la Compagnie de la Baie d'Hudson. Voir Newman, op. cit., p. 333-334.

18. Trad. «...eut-il agit de manière appropriée, il (de la Fontaine) pourrait encore posséder ce Poste, mais cet automne 1761 en route vers l'autre Poste de son Montagamiou, sa conduite a confirmé toute les choses mauvaises que $j$ 'ai entendues à son sujet et à la place d'être son Protecteur, je l'ai considéré comme un ennemi de l'État. M. Isbister dans sa lettre de mars 1762 montre combien j'ai été fautif d'entretenir des rapports avec cet homme. Le comportement de M. Lafontaine et l'Intelligence que nous avons que cet hiver des Privés et Pirates ont trouvé refuge et subsistance au Poste et dans la Baie-des-Chaleurs, me font estimer hautement nécessaire, à la demande de tous les marchands d'ici, d'armer un navire pour la protection de leur commerce...».

19. Dans Ivanhoe Caron, ptre.; «Les habitants du côteau Sainte-Geneviève», Bulletin des Recherches Historiques, vol. XXVII, p. 173.

20. D’après Jules Bélanger, Marc Desjardins et Yves Frenette, Histoire de la Gaspésie, Boréal Express/Institut Québécois de Recherche sur la Culture (IQRC), 1981, p. 164; à partir de 1760 la plupart des seigneuries créées sous le Régime français passent sous le contrôle de marchands, fonctionnaires ou militaires anglais: ainsi en mars 1772, la seigneurie de Grande-Rivière est concédée aux marchands Duncan Anderson et William Smith par un négociant de Québec, Henry Morin; en 1765 le général Frederick Haldimand acquierait celle de Pabos et en 1781, Donald McKinnon, ancien lieutenant de milice, celle de Matane. Amorcé en mars 1974, le projet d'une «histoire générale de la région», n'aurait «pu être mené à terme (...) [que] grâce à la participation, à compter de décembre 1980, de l'Institut..., alors que ce demier était inscrit dans un plus vaste projet d'histoire régionale, dirigé par Fernand Harvey» (p.9). Professeur de sociologie à l'Université du Québec à Rimouski de 1974 à 1980, F. Harvey y fondait en 1974 avec trois autres personnes, le Groupe de Recherche Interdisciplinaire en développement de l'est du Québec (GRIDEQ). Créé par une loi sanctionnée le 22 juin 1979, l'IQRC allait être présidé par Femand Dumont assisté d'un directeur général et d'un conseil de quatre membres nommés par le gouvernement. Dans Le Soleil du 8 décembre 1979, le joumaliste Raymond Giroux écrivait au sujet de l'organisme: «A long terme, il devra rechercher les courants profonds et les sources de la culture québécoise, analyser son évolution, étudier des voies d'accès possible et ainsi de suite.» 
21. D'après RJW, «Paspébiac Letterbooks», Archives publiques du Canada, Microfilm rell M903 (1790-1834), reproduit dans Lepage, ibid, Appendice I, p. 374.

22. Ibid, p. 375. Trad. «Un certain M. de la Fontaine est en négociation avec le propriétaire de la seigneurie de Grande-Rivière (...) mais le propriétaire ne voudra rien entendre si je ne garantie pas le payment (...) De la Fontaine s'est toumé vers moi (...) mais si vous suivez mon conseil nous devrions l'acheter immédiatement (...) Aller dans le sens de la Fontaine nous nuirait considérablement. J'ai stoppé l'affaire pour cet automne en lui disant que je dois vous consulter à ce sujet et que je lui ferai connaître le résultat le printemps prochain. Aussi devriez-vous m'écrire, en français, que vous ne pouvez avancer la somme...»

23. Thèses présentées dans Boyer et Lafontaine, op. cit., et élaborées dans D. Lafontaine, «L'organisation psychique-langagière comme mécanisme génératif central», in Psychanalyse et changement social, Actes et Instruments de la recherche en développement régional, GRIDEQ/Université du Québec à Rimouski, 1985, pp. 183-333. Cette OPL serait-elle la région médiane entrevue par Foucault?

24. Voir D. Lafontaine, «Les sociologues sans la (Société)», Cahiers de l'ACFAS, 33, 1985, pp. 69-84. 